\title{
MiR-125b inhibits cardiomyocyte apoptosis by targeting BAK1 in heart failure
}

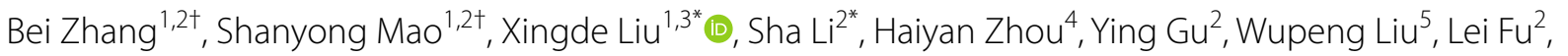
Chunyan Liao ${ }^{2}$ and Pengzhen Wang ${ }^{2}$

\begin{abstract}
Background: Although miR-125b plays a crucial role in many human cancers. However, its function in heart failure (HF) remains unclear. Our study aimed to investigate its involvement in heart failure.

Methods: In this study, the mouse HF model was successfully constructed through transverse aortic constriction (TAC) operation. Changes in mRNA and protein levels in isolated myocytes and heart tissues were examined using qRT-PCR, Western blot and Immunohistochemical staining and immunofluorescent staining. Changes in cardiac functions were examined using ultrasound. Interactions between miR-125b and BAK1 was analyzed using the luciferase reporter assay. Cardiomyocyte apoptosis was evaluated using the TUNEL staining.

Results: We found that miR-125b expression was significantly downregulated in myocardial tissues of HF mice. Moreover, miR-125b upregulation in HF mice injected with agomir-125b efficiently ameliorated cardiac function. Further, miR-125b upregulation significantly decreased the protein levels of apoptosis-related makers c-caspase 3 and Bax, while increased Bcl-2 expression. In addition, BAK1 was identified as a direct target of miR-125b. As expected, BAK1 overexpression observably reversed the effect of agomir-125b on cardiac function and on the expression of apoptosis-related makers in the heart tissues of HF mice.

Conclusions: Taken together, miR-125b overexpression efficiently attenuated cardiac function injury of HF mice by targeting BAK1 through inhibiting cardiomyocyte apoptosis, suggesting that miR-125b/BAK1 axis might be a potential target for the diagnosis or treatment of HF.
\end{abstract}

Keywords: MiR-125b, BAK1, Heart failure, Apoptosis

\section{Introduction}

Heart failure (HF) has become an epidemic disease affecting approximately $1 \%$ to $2 \%$ of the adult population worldwide (Tanai and Frantz 2015). HF is a complex disease and may occur due to many reasons including high blood pressure, aging, diabetes,

\footnotetext{
*Correspondence: xingdeliuguizhou6@163.com; iy3514@163.com ${ }^{\dagger}$ Bei Zhang and Shanyong Mao contributed equally to this work ${ }^{1}$ Guizhou Medical University, No. 9 Beijing Road, Yunyan District, Guiyang, Guizhou 550004, People's Republic of China

${ }^{2}$ Department of Ultrasound Medicine, The Affiliated Hospital of Guizhou Medical University, No. 28 Guiyi Street, Yunyan District, Guiyang, Guizhou 550004, People's Republic of China

Full list of author information is available at the end of the article
}

coronary heart diseases, and obesity (Lakatta 2002; Burchfield et al. 2013). In the last decades, several treatments such as pacing and defibrillation therapies, heart transplantation or mechanically assisted circulatory support have been widely applied for HF (Abdo 2017; Martinelli et al. 2011). However, HF is still common in adults accounting for substantial morbidity and mortality worldwide, and its prevalence is increasing (Metra and Teerlink 2017). Advances in the molecular mechanisms underlying the HF pathophysiology and treatment have led to the decreased incidence of patients with HF and the quality of life of the patients (Mazurek and Jessup 2017). Therefore, better understanding the specific mechanisms involved in HF will 
contribute to identifying new targets and even extend the window of treatment for HF.

MicroRNAs (miRNAs) are a group of small RNA molecules with approximately 22 nucleotides in length and play crucial roles in gene silencing and translational repression through directly binding to mRNAs of targets in human diseases (Vishnoi and Rani 2017; Batool et al. 2018; Zhou et al. 2016). Recently, many miRNAs are increasingly recognized to play essential roles in cardiovascular diseases including heart failure, specifically cardiac fibrosis and cardiomyocyte apoptosis (Vegter et al. 2016). MiR-125b has been identified to be closely associated with various biological processes in multiple diseases like nasopharyngeal carcinoma, acute myeloid leukemia and systemic lupus erythematosus (Li et al. 2017; Liu et al. 2017; Cao et al. 2018 Nagpal et al. found that miR-125b is essential for the transition from fibroblast to myofibroblast and cardiac fibrosis (Nagpal et al. 2016; Bie et al. 2016). However, to our best knowledge, its function and specific mechanisms in HF remain unclear.

Bcl-2 homologous antagonist/killer (BAK1), a proapoptotic factor, has been identified to play crucial roles in the apoptotic process of human cancer cells or mitochondrial cells in response to various stimulations. For example, BAK1 overexpression significantly induced mitochondrial apoptosis, while 4,4'-diisothiocyanostilbene-2,2'-disulfonic acid (DIDS) inhibited BAK1 overexpression-induced apoptosis via GSK3 $\beta / \beta$ catenin signaling pathway (Yang et al. 2018). Costunolide induces mitochondria-mediated apoptosis in human gastric adenocarcinoma BGC-823 cells through upregulating BAK1 expression (Yan et al. 2019). In addition, BAK1 has shown essential functions in cardiovascular diseases such as acute myocardial infarction. miRNA-125-5p could protect the heart against acute myocardial infarction by inhibiting the expression of pro-apoptotic factors BAK1 and KLF13 in cardiomyocytes (Bayoumi et al. 2018a). However, the underlying regulatory network of BAK1 in HF has not been studied in detail.

In this study, we first established the HF mouse model through TAC operation and found that miR$125 \mathrm{~b}$ was significantly downregulated in heart tissues of HF mice. Further, a series of in vitro and in vivo experiments all confirmed that miR-125b overexpression efficiently alleviate cardiac functional injury in HF mice through directly targeting BAK1, thereby suppressing cardiomyocyte apoptosis, and suggested that miR-125b/BAK1 axis might be a potential target for the diagnosis and treatment of HF.

\section{Materials and methods Animal model}

A total of $40 \mathrm{C} 57 \mathrm{BL} / 6$ mice (male, 8-10 weeks old, approximately $22-25 \mathrm{~g}$ ) were obtained from Guizhou Medical University and kept at $20 \pm 2{ }^{\circ} \mathrm{C}$ in a room with $12 \mathrm{~h}$ light/dark cycle and approximately $50-60 \%$ humidity. To construct HF mouse model, mice were fasted for $12 \mathrm{~h}$, depilated, anesthetized with $4 \%$ chloral hydrate $(0.1 \mathrm{ml} / 10 \mathrm{~g})$, and subjected to transverse aortic constriction (TAC) operation as previously described ( $\mathrm{Li}$ et al. 2018a). Briefly, following intubation and mechanical ventilation of the mice, the transverse aorta was accessed through partial thoracotomy via the upper edge of the sternum. The aortic arch was separated and ligated with a No.27 needle using 7-0 nylon threads. Then the needle was removed to cause $60 \%$ annular coarctation of the aortic arch. After the operation, the chest of mice was closed, the wound was sutured. All animal experiments were performed according to the guideline of Guizhou Medical University. The study was approved by Guizhou Medical University.

\section{Animal grouping and treatment}

The miRNA reagents, mir-125b agomir (agomir-125b) and scrambled control (NC agomir) were purchased from Shanghai GenePharma Co., Ltd. (Shanghai, China) and mixed with linear polyethyleneimine (PEI) nanoparticles (Sigma-Aldrich, St. Louis, MO) for intravenous injection. The construction of RNA interference plasmid of BAK1 (sh-BAK1) was completed by Shanghai Meixuan Biological Science and Technology Co., LTD. Empty pcDNA3.1 vector (Invitrogen, Shanghai, China) was used to construct BAK1 expression vector by Sangon (Shanghai, China). Sh-NC or empty pcDNA3.1 vector was used as the $\mathrm{NC}$ group.

Mice were randomly divided into ten groups $(n=8)$ : (1) TAC group (mice were treated with TAC), (2) $\mathrm{TAC}+$ agomir-NC group (TAC mice were injected with agomir-125b negative control through the tail vein), (3) TAC + agomir-125b (TAC mice were injected with agomir-125b through the tail vein), (4) agomir-125b + sh-NC group (TAC mice were injected with agomir-125b and sh-NC through the tail vein), (5) agomir- $125 \mathrm{~b}+$ shBAK1 group (TAC mice were injected with agomir-125b and sh-BAK1 through the tail vein), (6) control group (mice without any treatment), (7) OE-BAK1 group (normal mice were injected with OE-BAK1 to overexpress BAK1), (8) OE-NC group (normal mice were injected with OE-NC), T9) AC + agomir-125b + OE-BAK1 (TAC mice were injected with agomir-125b and OE-BAK1), and (10) TAC + agomir-125b + OE-NC (TAC mice were injected with agomir-125b and OE-NC). For the sham 
mice, the steps were the same expecting the aortic arch was not ligated. Mice were sacrificed at 10 weeks of operation by intraperitoneal injection of excessive pentobarbital $(100-150 \mathrm{mg} / \mathrm{kg})$. The heart tissues of mice were harvested and used for the subsequent experiments. The sequences were 5'-UCCCUGAGACCCUAACUU GUGA-3' for Agomir-125b, 5'-UUUGUACUACACAAA AGUACUG-3' for Agomir-NC, 5'-AAAC GUAGCU UCGAAAGACCU for shBAK1, and 5'-GTTCTCCGA ACGTGTCACGT-3' for shNC.

\section{The detection of cardiac functions by ultrasound}

The structure and function of mouse hearts were detected according to previous reports (Zhao et al. 2011; Jiang et al. 2014; Zhao et al. 2017) using the VeVo 770 high-resolution small animal ultrasound system. After mice were anesthetized by inhaling $2 \%$ isoflurane, the ultrasonic cardiogram of the limb leads of the mice was measured by using a $15 \mathrm{~mm}$ deep, $30 \mathrm{MHz}$ probe. Twodimensional guided $\mathrm{M}$-mode tracings were recorded in both parasternal long and short axis views at the level of papillary muscles. The mean left ventricular ejection fraction (LVEF), left ventricular fractional shortening, left ventricular septal thickness, left ventricular septal diastole (IVS; d), left ventricular posterior wall thickness; left ventricular posterior diastole (LVPW; d), and left ventricular mass (LV mass) were calculated with the established standard equations. All measurements were made from at least three beats and averaged.

\section{H\&E staining assay}

The H\&E staining assay was performed as previously described (Liu et al. 2018; Xu et al. 2019). Briefly, the heart tissues were collected, fixed with $4 \%$ paraformaldehyde at $4{ }^{\circ} \mathrm{C}$, dehydrated with gradient alcohol, cleared in xylene, embedded, and sliced into $4 \mu \mathrm{m}$ sections. Then the sections were dyed in hematoxylin, washed away with running water, and dyed with $1 \%$ eosin. After washing with running water again, the sections were dehydrated, cleared, blocked with neutral gum, and dried for three days. Finally, heart tissues were photographed under a light microscope in $200 \times$ magnification. More than 100 myocytes in the sections were outlined in each group.

\section{Masson staining assay}

The Masson staining assay was performed by using a Masson Stain Kit according to the instructions. The level of myocardial fibrosis was evaluated by calculating the percentage of myocardial tissue fibrosis or the blue region (collagen). For fibrosis measurements, $>25$ fields were measured in each group.

\section{TUNEL staining assay}

To evaluate the apoptosis rate of cardiomyocytes in mouse heart tissues, TUNEL staining was performed using the TUNEL BrightGreen Apoptosis Detection Kit according to the instructions. Of which, apoptotic nuclei were stained with green fluorescein and total cardiomyocyte nuclei were stained with DAPI. Representative images were captured using a confocal microscope and the apoptosis rate was calculated as TUNEL positive nuclei/DAPI-stained nuclei $\times 100 \%$.

\section{Immunohistochemical staining assay}

Immunohistochemistry staining was performed on deparaffinized sections to show collagen protein retention. After dewaxing and hydrating, the nonspecific antigens of the specimens were blocked with $3 \% \mathrm{H}_{2} \mathrm{O}_{2}$ for $15 \mathrm{~min}$ and with $5 \%$ bovine serum albumin (BSA) (Beyotime, China) in phosphate buffer solution (PBS) for $45 \mathrm{~min}$ at $37^{\circ} \mathrm{C}$, respectively. Then, the specimens were incubated with primary antibodies against Col I (ab260043, 1:400, Abcam) and Col III (ab7778, 1:200, Abcam) at $4{ }^{\circ} \mathrm{C}$ for $12 \mathrm{~h}$. After washed three times with PBS to remove the redundant primary antibodies, the specimens were incubated with tagged secondary goat anti-mouse $\operatorname{lgG}$ or goat anti-rabbit lgG (ZSGB-Bio, Beijing, China) at $37{ }^{\circ} \mathrm{C}$ for $60 \mathrm{~min}$. Finally, the immunohistochemistry staining was stopped with $\mathrm{DAB}$ chromogen kit (ZSGB-BIO, Beijing, China) followed by hematoxylin staining, and the positive-staining was observed using a fluorescence microscope (Nikon, Japan).

\section{Immunofluorescent staining assay}

Immunofluorescence staining was performed on deparaffinized sections. After dewaxed and hydrated, the nonspecific antigens of the specimens were blocked with $3 \% \mathrm{H}_{2} \mathrm{O}_{2}$ for $15 \mathrm{~min}$ and with $5 \%$ bovine serum albumin (BSA) (Beyotime) in PBS for $45 \mathrm{~min}$ at $37^{\circ} \mathrm{C}$, respectively. Subsequently, the sections were incubated with primary anti-Cas-3 (ab32351, 1:200, Abcam) and anti$\alpha$-sarcomeric actin (ab68167, 1:200, Abcam) antibodies at $4{ }^{\circ} \mathrm{C}$ overnight followed by goat anti-rabbit IgG Alexa FluorVR 647 (ab150083, 1:1500, Abcam, Cambridge, United Kingdom). Nuclei were stained using DAPI (Beyotime, China). Angiogenesis was observed using a fluorescence microscope (Nikon, Japan).

\section{Extraction of primary cardiomyocytes}

Mice were sacrificed at 10 weeks of operation by intraperitoneal injection of excessive pentobarbital (100$150 \mathrm{mg} / \mathrm{kg}$ ) and immersed in 70\% ethanol for $1 \mathrm{~min}$. The hearts were removed and placed in PBS. The atrium and excess arterial tissues were cut off. Ventricular 
tissues were chopped and transferred into a $15 \mathrm{ml}$ centrifuge tube. A disinfectant solution composed of $0.125 \%$ trypsin, $0.1 \%$ collagenase II and $0.05 \%$ DNase was added. After incubation at $37^{\circ} \mathrm{C}$, the cell suspension was collected by centrifuge. The process was repeated for 3 times. The collected suspension was mixed with high glucose DMEM supplemented with $10 \%$ FBS, $1 \%$ penicillin and $1 \%$ streptomycin. After pipetting up and down several times, fibroblasts were filtered with a 230-mesh screen and separated using the differential attachment method at $60 \mathrm{~min}$ each time and placed at $37^{\circ} \mathrm{C}$ in a three-gas incubator. Unattached primary cardiomyocytes were collected for Western blot.

\section{qRT-PCR}

The total RNA was extracted from $60 \mathrm{mg}$ heart tissues using the TRIzol reagent (Invitrogen, Carlsbad, USA) according to the instructions. After DNase I treatment, RNA was reverse transcribed into cDNA using with PrimeScript Reverse Transcriptase (TaKaRa, Japan) according to the manufacturer's instruction and subjected to quantitative real-time PCR on LightCycler 480 (Roche, Basel, Switzerland). The relative expression changes of targets were analyzed by the $2^{-\Delta \Delta C T}$ method, with GAPDH and U6 as the internal reference. The primers using in this study were mouse-ANP forward ACCACC TGGAGGAGAAGA and reverse TTCAAGAGGGCA GATC TATC, mouse-BNP forward TCCAGCAGAGAC CTCAAAATTCC and reverse TCAAAGGTGGTCCCA GAGCT, mouse- $\beta$-MHC forward CCGAGTCCCAGG TCA ACAA and reverse CTTCACGGGCACCCTTGG A, mouse-GAPDH forward ACTCCACTCAC GGC AAATTC and reverse TCTCCATGGTGGTGAAGACA, miR-125b forward TCCCTGAGACCCTAACTTGTGA and reverse AGTCTCAGGGTCC GAGGTATTC, and U6 forward CTCGCTTCGGCAGCACA and reverse AACGC TTCACGAATTTGCGT.

\section{Western blot}

Total proteins of heart tissues were isolated by using RIAP lysis buffer (Beyotime, China) according to the instructions, and protein concentrations were measured using the BCA Protein Assay Kit (Abcam, USA). Approximately equal amounts of proteins were separated by $12 \%$ SDS-PAGE and then transferred onto the PVDF membranes (Millipore, USA). After blocking by TBST containing 5\% skim milk for $2 \mathrm{~h}$ at room temperature, the membranes were incubated with primary antibodies against BAK1 (Abcam, ab104124, 1:1000), C-caspase 3 (Abcam, ab2302, 1:300), Bax (Abcam, ab182733, 1:300) and Bcl-2 (Abcam, ab194583, 1:300) overnight at $4{ }^{\circ} \mathrm{C}$, with GAPDH (Abcam, GAPDH,1:1000) as the internal reference. After washing, the membranes were exposed to horseradish peroxidase-labeled secondary antibody (Thermo Fisher Scientific, 1:200) for 2 h. Signals were detected by using the ECL kit and visualized by ChemiDoc $^{\mathrm{TM}}$ MP System (Bio-Rad). The gray value of targets was analyzed with Image Lab ${ }^{\mathrm{TM}}$ Software. The protein expression level was normalized to GAPDH on the same PVDF membrane.

\section{Luciferase reporter assay}

To determine the correlation between miR-125b and BAK1, the fragments containing wild type (WT) or mutant type (MUT) 3' UTR of BAK1 with the putative miR-125b binding sites were amplified and cloned into pmiR-RB-Report vector. The luciferase reporter plasmids were co-transfected with miR-125b mimics or miR-NC into HEK293T cells using Lipofectamine 2000 according to the manufacturer's instructions. After transfection for $48 \mathrm{~h}$, the relative Renilla luciferase activities were measured using Dual-Luciferase Reporter Assay System.

\section{Statistical analysis}

Data were analyzed using GraphPad Prism 7.0 and presented as mean \pm standard deviation (SD). Each experiment was repeated at least three times. After verifying data normality, the difference between two groups was determined by using the $t$ test and among multiple groups was determined by one-way ANOVA in SPSS 19.0. $\mathrm{P}<0.05$ was considered significant.

\section{Results}

MiR-125b was significantly downregulated in heart tissues of HF mice

First, the HF mice model was established through TAC induction, and the heart function was evaluated ten weeks after the operation. The results showed that the mRNA expression of ANP, BNP and $\beta$-MHC was markedly upregulated in the heart tissues of TAC mice compared with that of the sham group $(\mathrm{p}<0.001)$ (Fig. 1A). Meanwhile, compared with the sham group, the left ventricular ejection fraction $(\mathrm{p}<0.01)$ (Fig. $1 \mathrm{~B}$ and $\mathrm{C})$ and left ventricular fractional shortening $(\mathrm{p}<0.01)$ (Fig. 1D) was significantly decreased in the heart tissues of TAC mice. By contrast, left ventricular septal thickness diastole (IVS; d) $(\mathrm{p}<0.05)$ (Fig. 1E) and left ventricular posterior wall thickness diastole (LVPW; d) $(\mathrm{p}<0.05)$ (Fig. 1F) were increased in the heart tissues of TAC mice compared with those of the sham group. Similarly, compared with the sham group, the ratio of left ventricular mass/ body weight (LV mass/BW) was also increased in the heart tissues of TAC mice $(\mathrm{p}<0.05)$ (Fig. 1G). These data suggested that the HF mice model was successfully established ten weeks after TAC operation and could be used for the subsequent analysis. Then miR-125b expression 


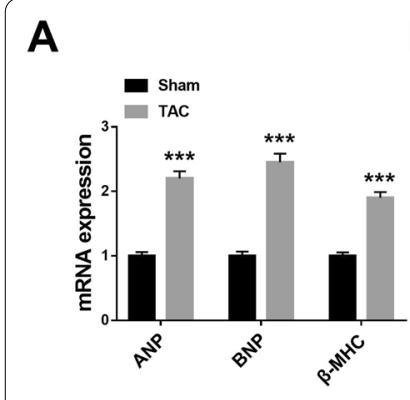

E

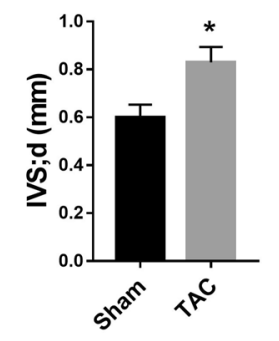

B

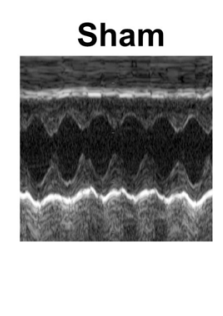

F

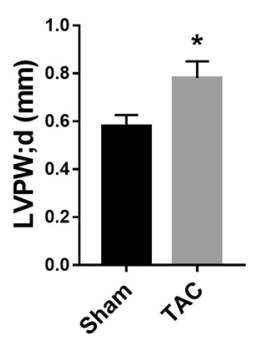

G

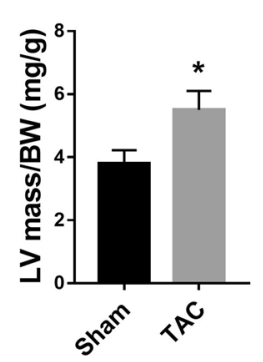

C

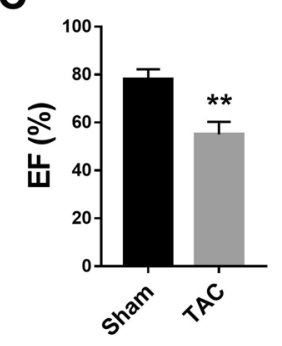

H

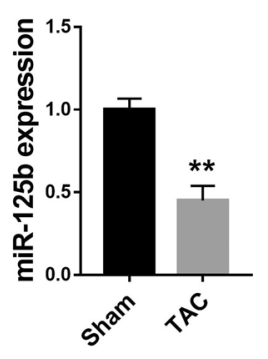

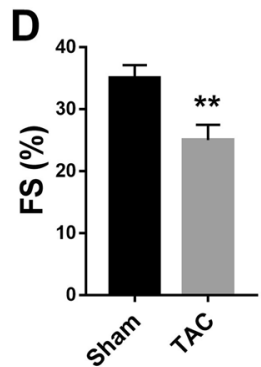

I

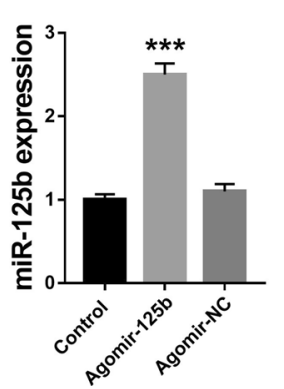

Fig. 1 MiR-125b was downregulated in the heart tissues of HF mice. Mice were treated with or without TAC, and the structure and function of mice hearts were evaluated ten weeks after TAC operation by high resolution small animal ultrasound system and analyzed by M mode. A The mRNA levels of ANP, BNP and $\beta-M H C$ were evaluated by qRT-PCR $(n=5)$. B Representative images of echocardiographic measurements of mice hearts $(n=8)$. C Left ventricular ejection fraction (EF\%) $(n=8)$. D Left ventricular fractional shortening $(F S \%)(n=8)$. E Left ventricular septal thickness; diastole (IVS; d) ( $n=8)$. F Left ventricular posterior wall thickness; diastole, (LVPW; $d)(n=8)$. G Left ventricular mass/body weight (LV mass/BW) $(n=8)$. H The mRNA level of miR-125b was evaluated by qRT-PCR $(n=5)$. I Agomir-125b or agomir-NC was injected into mice, and the mRNA level of miR-125b was evaluated by qRT-PCR $(n=5) .{ }^{*} p<0.05,{ }^{* *} p<0.01,{ }^{* * *} p<0.001$ vs. sham group or control group

was evaluated, and the results indicated that miR-125b mRNA level was significantly downregulated in the heart tissues of TAC mice in comparison with the sham group $(\mathrm{p}<0.01)$ (Fig. 1H). Further, agomir-125b or agomir-NC was applied through the tail vein to overexpress miR$125 \mathrm{~b}$, and the efficiency was evaluated by qRT-PCR assay. The results showed that agomir-125b significantly increased miR-125b expression compared with the agomir-NC group $(\mathrm{p}<0.001)$ (Fig. 1I). These results suggested that miR-125b plays a potential protective role for cardiac function during heart failure.

\section{MiR-125b overexpression alleviated cardiac function injury in HF mice}

To determine the role of miR-125b in heart tissues, agomir-125b was intravenously injected through tail into TAC mice to overexpress miR-125b. Compared with TAC mice, the mRNA expression of ANP $(\mathrm{p}<0.001)$ (Fig. 2A), $\mathrm{BNP}(\mathrm{p}<0.001)$ (Fig. 2B) and $\beta-\mathrm{MHC}(\mathrm{p}<0.01)$ (Fig. $2 \mathrm{C}$ ) in the heart tissues was all significantly decreased in TAC+agomir-125b group, while exhibited no obvious change in $\mathrm{TAC}+$ agomir-NC agomir-125b group. Meanwhile, compared with TAC group, the left ventricular ejection fraction $(\mathrm{p}<0.05)$ (Fig. $2 \mathrm{D}$ and $\mathrm{E}$ ) and left ventricular fractional shortening $(\mathrm{p}<0.05)$ (Fig. 2F) were increased, while left ventricular septal thickness diastole (IVS; d) (p<0.05) (Fig. 2G), left ventricular posterior wall thickness diastole (LVPW; d) $(\mathrm{p}<0.05)($ Fig. $2 \mathrm{H})$ and left ventricular mass/body weight $(\mathrm{LV}$ mass/BW) $(\mathrm{p}<0.05)$ (Fig. 2I) were decreased in TAC + agomir-125 group and showed no obvious change in $\mathrm{TAC}+$ agomir-NC group. Moreover, H\&E staining and Masson staining (Fig. 2J) were performed to evaluate the degree of myocardial damage. The data indicated that TAC treatment significantly increased the percentage of cross section in heart tissues compared with the sham group $(\mathrm{p}<0.001)$, while miR-125b overexpression significantly decreased the effect of TAC effect on cross section ( $\mathrm{p}<0.01$ ) (Fig. $2 \mathrm{~K}$ ). Furthermore, TAC treatment significantly increased the percentage of myocardial tissue fibrosis compared with the sham group $(\mathrm{p}<0.001)$, while miR-125b overexpression significantly decreased the effect of TAC on myocardial tissue fibrosis $(\mathrm{p}<0.001)$ (Fig. 2L). Additionally, about $80 \%$ of the myocardial collagen fibers were Col I, which can maintain the strength of the ventricular wall due to its great hardness and strong anti-pull function, and about $11 \%$ were Col III, which has relatively fine fibrosis and good extensibility and elasticity (Bodh et al. 1995; 


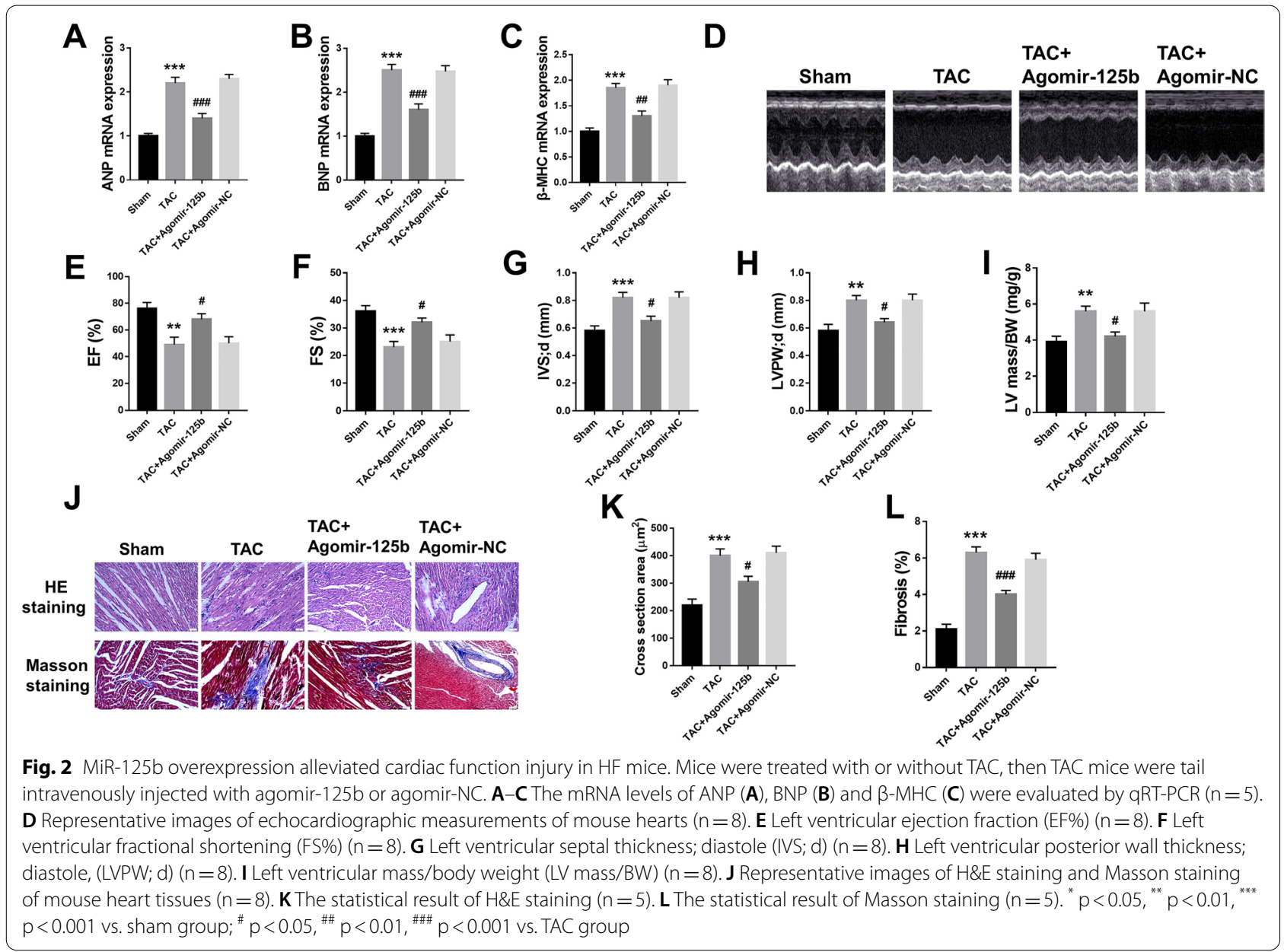

Hanna et al. 2021). The ratio of these two collagens is of great significance for maintaining the normal structure of cardiac tissue and the integrity of cardiac function (Bodh et al. 1995; Hanna et al. 2021). We then demonstrated that miR-125b overexpression alleviated Col I expression in TAC-induced myocardial tissues and promoted Col III expression (Additional file 1: Fig. S1). These results indicated that miR-125b overexpression efficiently attenuated TAC-induced cardiac function injury.

\section{MiR-125b overexpression inhibited cardiomyocyte apoptosis in HF mice}

To explore whether miR-125b affects the heart failure through modulating the process of cardiomyocyte apoptosis, the expression of apoptosis-related proteins in heart tissues was detected by Western blot. The results showed that TAC treatment significantly increased the protein expression of $C$-caspase $3(\mathrm{p}<0.001)$ (Fig. 3A and B) and $\operatorname{Bax}(\mathrm{p}<0.001)$ (Fig. 3A and D) compared with the sham group and decreased Bcl-2 expression $(\mathrm{p}<0.001)$ (Fig. $3 \mathrm{~A}$ and $\mathrm{C}$ ), while miR-125b overexpression significantly reversed the effect of TAC operation on the expression of C-caspase $3, \mathrm{Bcl}-2$ and $\operatorname{Bax}(\mathrm{p}<0.01$, $\mathrm{p}<0.001$ ) (Fig. 3A-D). Meanwhile, the apoptosis of cardiomyocyte was evaluated by TUNEL staining (Fig. 3E), and the results indicated that TAC treatment significantly increased the percentage of TUNEL positive cells compared with the sham group $(\mathrm{p}<0.01)$, while miR-125b overexpression significantly decreased the percentage of TAC-induced TUNEL positive cells compared with agomir-NC group $(\mathrm{p}<0.05)$ (Fig. 3F). To determine the origin of these apoptotic markers from cardiomyocytes, we performed immunofluorescence staining (Additional file 2: Fig. S2A) and Western blot (Additional file 2: Fig. S2B) fto examined the levels of both Cas-3 and myocyte marker $\alpha$-Sarcomeric actin. The results revealed that miR-125b overexpression efficiently inhibited cardiomyocyte apoptosis in HF mice.

\section{BAK1 was a target of miR-125b}

Next, the Targetscan database was used to predict the potential targets of miR-125b, and the results showed that 

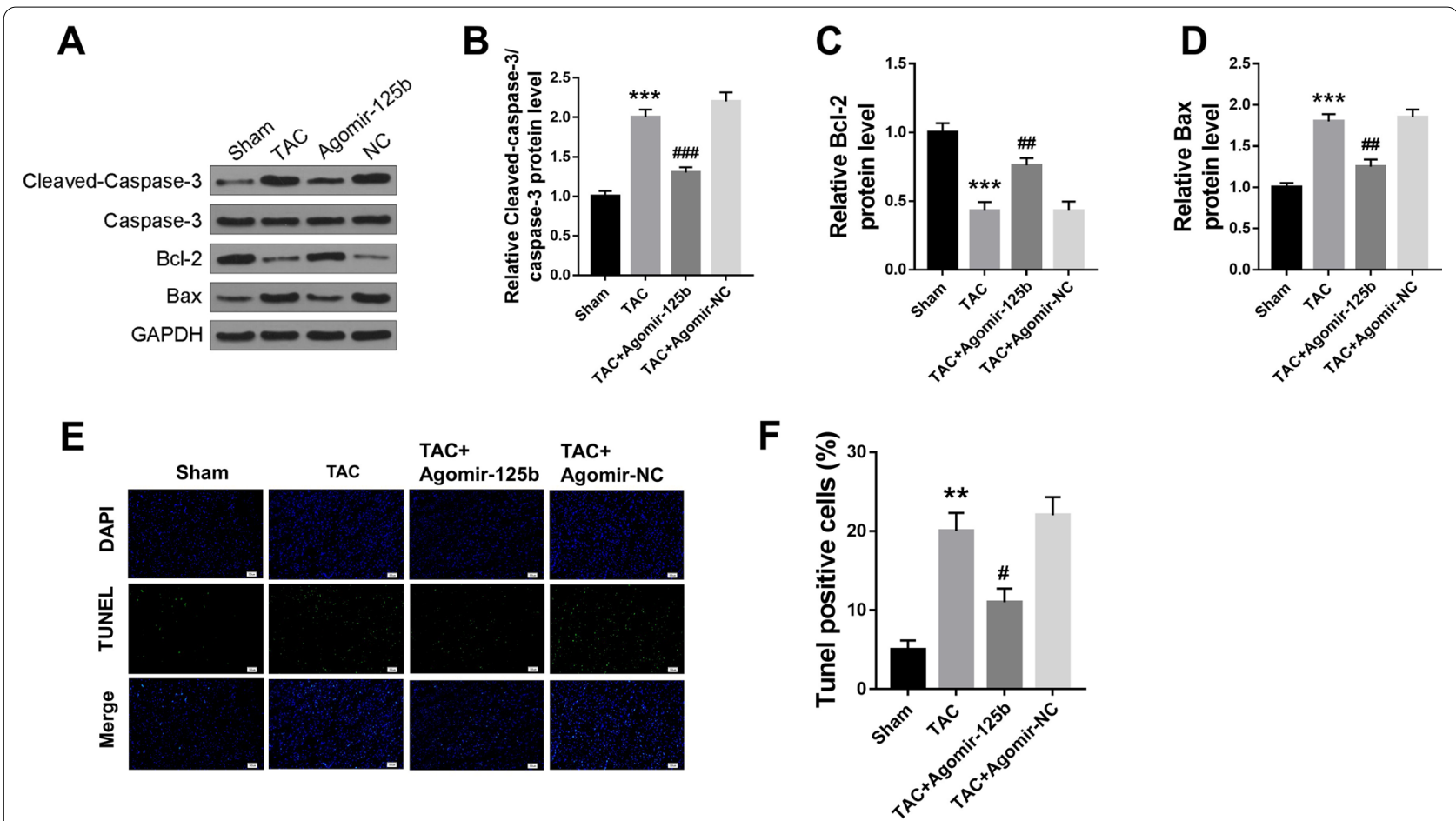

Fig. 3 MiR-125b overexpression inhibited cardiomyocyte apoptosis in HF mice. Mice were treated with or without TAC. TAC mice were tail intravenously injected with agomir-125b or agomir-NC. A The protein expression of C-caspase 3, caspase 3, Bcl-2 and Bax in heart tissues was detected by Western blot $(n=5)$. B-D The relative expression changes in C-caspase $3(\mathbf{B}), \mathrm{BCl}-2(\mathbf{C})$ and Bax (D) were analyzed by Image Lab ${ }^{\mathrm{TM}}$ Software $(n=5)$. E Representative images of TUNEL staining of heart tissues by confocal microscopy $(n=8)$. $\mathbf{F}$ The apoptosis rate was calculated as the ratio of TUNEL positive nuclei to DAPI-stained nuclei $(n=5) .{ }^{*} p<0.05,{ }^{* *} p<0.01,{ }^{* * *} p<0.001$ vs. sham group; ${ }^{\#} p<0.05,{ }^{\# \#} p<0.01,{ }^{\# \# \#} p<0.001$ vs. TAC group

there was a putative binding site between miR-125b and $3^{\prime}$ UTR of BAK1 (Fig. 4A). Then the fragments containing WT or MUT $3^{\prime}$ UTR of BAK1 were co-transfected with miR-125b mimics or miR-NC into HEK293T cells, and the luciferase reporter assay was performed. The results indicated that miR-125b mimics significantly decreased the relative luciferase activity of WT $3^{\prime}$ UTR of BAK1 $(\mathrm{p}<0.001)$, while showed no obvious change of the MUT 3' UTR of BAK1 (Fig. 4B). Further, agomir-125b or agomir-NC was tail intravenously injected into normal mice, and the results indicated that miR-125b overexpression significantly decreased BAK1 protein level in heart tissues compared with the agomir-NC group $(\mathrm{p}<0.01)$ (Fig. $4 \mathrm{C}$ ). In addition, agomir-125b or agomir-NC was tail intravenously injected into TAC mice, and the results indicated that TAC treatment markedly increased BAK1 protein level compared with the sham group $(\mathrm{p}<0.001)$, and miR-125b overexpression observably decreased TAC-induced BAK1 expression compared with the agomir-NC group $(\mathrm{p}<0.05)$ (Fig. 4D). Moreover, BAK1 overexpression vector (OE-BAK1) or negative control (OE-NC) was constructed and tail intravenously injected into normal mice, and the results indicated that BAK1 overexpression significantly increased BAK1 expression at both mRNA (p<0.001) (Fig. 4E) and protein $(\mathrm{p}<0.001)$ (Fig. 4F) levels in heart tissues of mice compared with the OE-NC group. These results suggested that BAK1 was a direct target of miR-125b.

\section{BAK1 overexpression significantly reversed} agomir-125b-induced protective effects in heart failure

To determine whether the protective effect of miR-125b overexpression was mediated by BAK1, TAC mice were co-transfected with agomir-125b and OE-BAK1, or with agomir-125b and OE-NC, and the heart function was evaluated. Co-transfection with agomir-125b and OEBAK1 significantly reversed agomir-125b-induced protective effect in TAC mice on the mRNA level of ANP $(\mathrm{p}<0.01)$ (Fig. 5A), BNP ( $<$ 0.001) (Fig. 5B) and $\beta$-MHC $(\mathrm{p}<0.001)$ (Fig. 5C), the left ventricular ejection fraction $(\mathrm{p}<0.05)$ (Fig. 5D and E), left ventricular fractional shortening $(\mathrm{p}<0.05)$ (Fig. 5F), left ventricular septal thickness diastole (IVS; d) ( $<<0.05)$ (Fig. 5G), left ventricular posterior wall thickness diastole (LVPW; d) $(\mathrm{p}<0.05)($ Fig. 5H) and left ventricular mass/body weight (LV mass/BW) $(\mathrm{p}<0.05)$ (Fig. 5I) compared with the agomir-125b and 

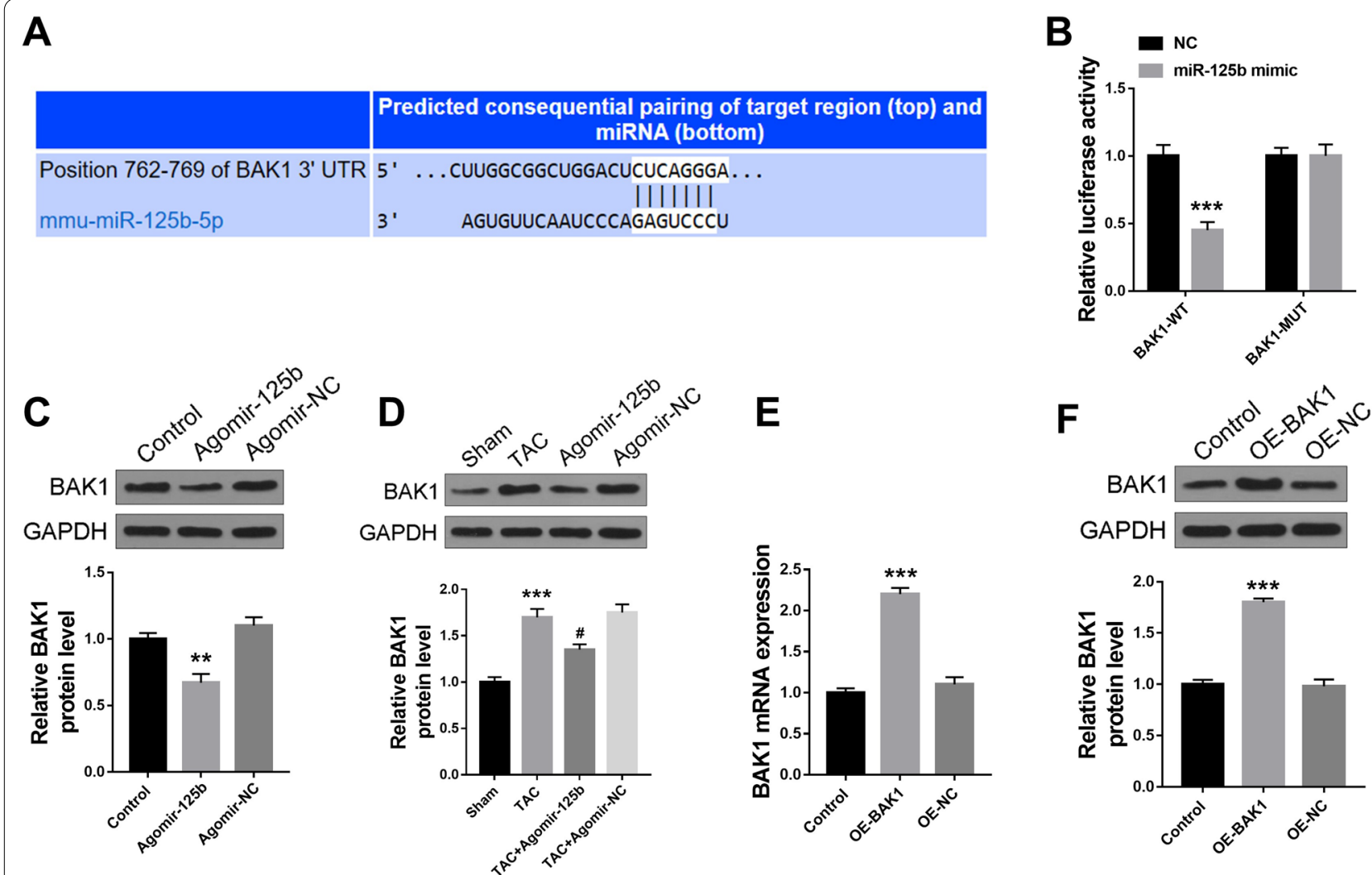

Fig. 4 BAK1 was a target of miR-125b. A The putative binding sites between miR-125b and 3' UTR of BAK1 was predicted by Targetscan database. B The relative luciferase activity in HEK293T cells was evaluated by dual luciferase report system $(n=6){ }^{* * *} p<0.001$ vs. miR-NC group. C Agomir-125b or agomir-NC was tail intravenously injected into normal mice, and the protein expression of BAK1 was evaluated by Western blot $(n=5)$. ${ }^{* *} p<0.01$, ${ }^{* * *} \mathrm{p}<0.001$ vs. control group. D Agomir-125b or agomir-NC was tail intravenously injected into TAC mice, and the protein expression of BAK1 was evaluated by Western blot $(n=5) .{ }^{* *} p<0.01,{ }^{* * *} p<0.001$ vs. sham group. $\mathbf{E}$ and $\mathbf{F}$ OE-BAK1 or OE-NC was tail intravenously injected into normal mice, then mRNA expression $(\mathbf{E})$ and protein expression $(\mathbf{F})$ was evaluated $(n=5) .{ }^{* * *} p<0.001$ vs. control group

OE-NC group. In addition, the H\&E staining and Masson staining (Fig. 5J) were performed to evaluate the degree of myocardial damage. The results indicated that co-transfection with agomir-125b and OE-BAK1 significantly increased the percentage of cross section $(\mathrm{p}<0.05)$ (Fig. 5K) and myocardial tissue fibrosis ( $\mathrm{p}<0.01)$ (Fig. 5L) in heart tissues compared with the agomir-125b and OE-NC group. We also demonstrated that OE-BAK1 overexpression reversed the effect of mir-125b on Col I and col III expression of TAC-induced myocardial tissue (Additional file 3: Fig. S3). These results demonstrated that BAK1 overexpression significantly reversed agomir125b-induced protective effect in heart failure.

\section{BAK1 overexpression significantly reversed agomir-125b-inhibited cardiomyocyte apoptosis in HF mice}

TAC mice were co-transfected with agomir-125b and OE-BAK1, or with agomir-125b and OE-NC, and the index of myocardial apoptosis was evaluated. The results indicated that co-transfection with agomir$125 \mathrm{~b}$ and OE-BAK1 significantly increased the protein expression of $\mathrm{C}$-caspase $3(\mathrm{p}<0.01)$ (Fig. 6A and $\mathrm{B})$ and $\mathrm{Bax}(\mathrm{p}<0.01)$ (Fig. 6A and $\mathrm{D})$ induced by agomir-125b, while decreased Bcl-2 expression $(\mathrm{p}<0.001)$ (Fig. 6A and B) compared with the agomir-125b and OE-NC group. In addition, cardiomyocyte apoptosis was evaluated by TUNEL staining (Fig. 6E), and the results showed that co-transfection with agomir-125b and OE-BAK1 significantly increased the percentage of TUNEL positive cardiomyocytes with miR-125b overexpression compared to those with agomir-125b and OE-NC overexpression $(\mathrm{p}<0.05)$ (Fig. 6F). We also further identified that apoptosis markers were derived from cardiomyocytes (Additional file 4: Fig. S4). These results revealed that BAK1 overexpression significantly reversed agomir-125b inhibited-cardiomyocyte apoptosis in HF mice. 


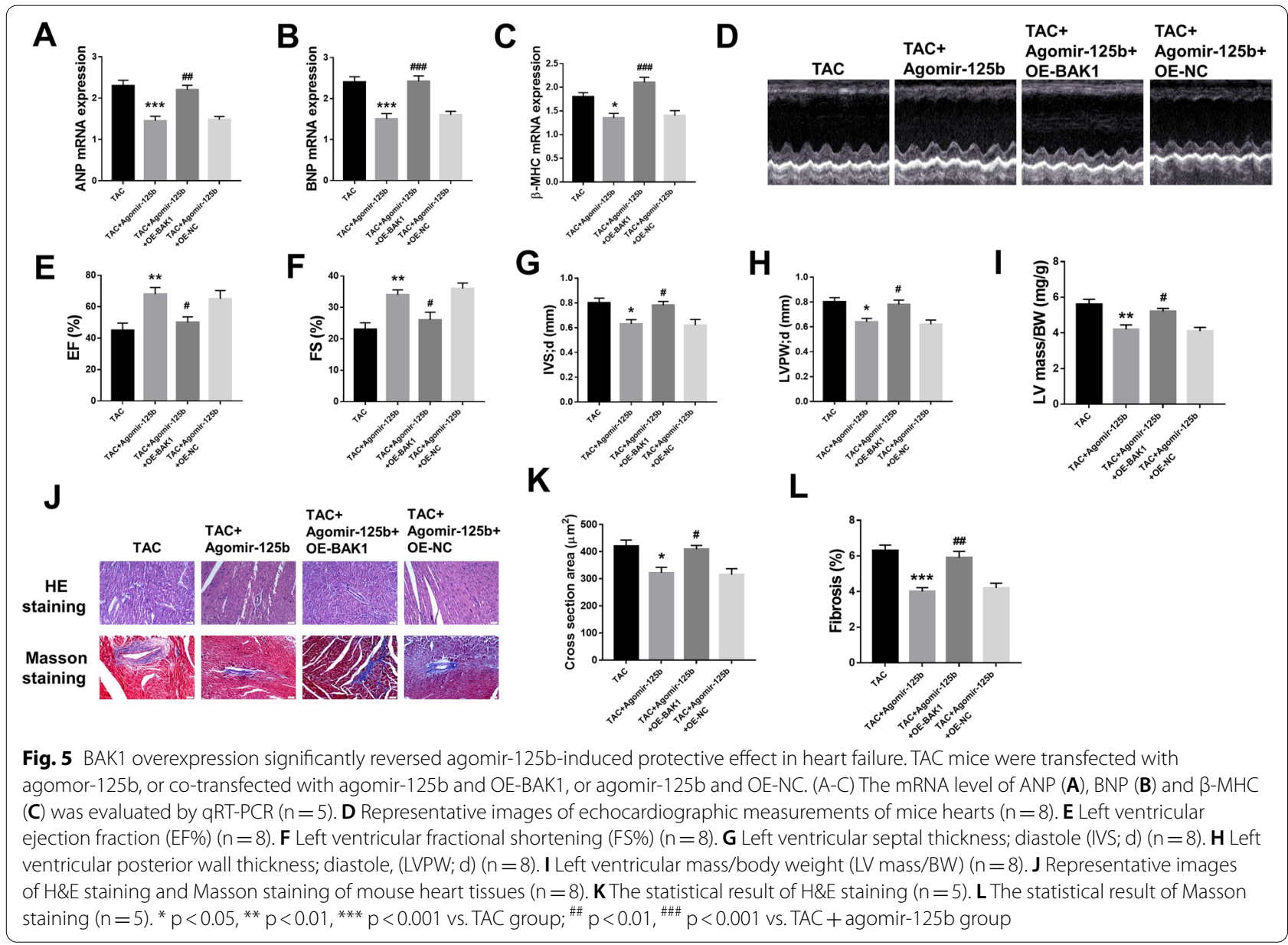

\section{Discussion}

HF is increasing in prevalence with a lack of efficient therapies that produce major severe effects on its associated mortality (McMurray and Pfeffer 2005). Recently, a large number of microRNAs have been identified to be closely associated with the progression of HF and might be considered as the potential targets for therapy (Shah et al. 2017; Qiao et al. 2019; Verjans et al. 1979). Wang et al. found that the heart-related circRNA (HRCR) protects the heart against pathological hypertrophy and HF through directly targeting miR-223 (Wang et al. 2016). Yang et al. found that miR-19b-1 reverses ischemiainduced heart failure through inhibiting cardiomyocyte apoptosis and targeting Bcl2-like 11/BIM (a pro-apoptotic gene of the Bcl-2 family) (Yang et al. 2019). Although miR-125b exerts broad effects in human diseases, the specific function of miR-125b in HF remains unclear and attracts our attention. In the present study, to explore the role and underlying mechanisms of miR-125b in detail, the HF mice model was successfully established by TAC operation. Interestingly, our results indicated that miR$125 \mathrm{~b}$ was significantly downregulated in the heart tissues of HF mice, suggesting a potential protective effect of miR-125b in HF.

Myocardial interstitial fibrosis is well known to result in left ventricular dysfunction, contributing to the development of HF (González et al. 2018). It has been reported that myocardial interstitial fibrosis is a major cause of cardiac dysfunction with adverse tissue remodeling (Schelbert et al. 2014; Butler et al. 2014; Davis and Molkentin 2014). Current therapies that improve HF survival primarily target the pathogenic mechanisms that occur within cardiomyocytes but not those that take place outside cardiomyocytes in the interstitial space that houses collagen and fibroblasts (Yang et al. 2016). This lack of treatment against interstitial mechanisms of HF might be one cause of mortality and morbidity for HF. Here, our results demonstrated that miR-125b overexpression efficiently inhibited TAC-induced myocardial tissue fibrosis. In addition, cardiomyocyte apoptosis also plays an important facilitation effect in the progression of HF. Inhibition of cardiomyocyte apoptosis can efficiently protect the heart against HF (Deng et al. 2019; Li et al. 2018b). Apoptosis-related markers such as C-caspase 


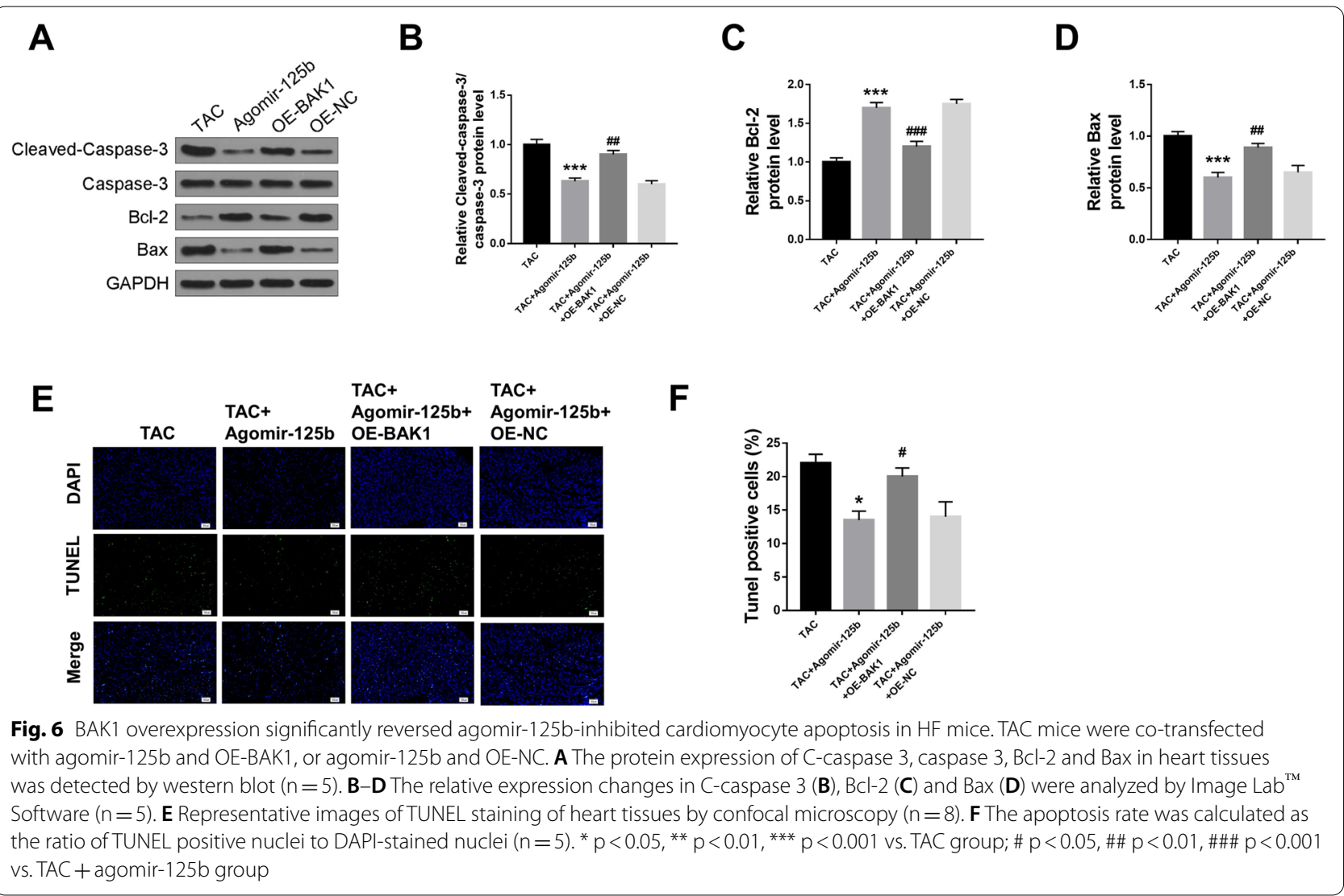

3, Bax and Bcl-2 are often used to represent the degree of cell apoptosis, of which, caspases (caspase-3, caspase-6, and caspase-8) are a family of cysteine proteases that mediate apoptosis induced by a variety of stimuli (Jin et al. 2009), Bax and Bcl-2 are the members of antiapoptotic Bcl-2 family, and Bax is a pro-apoptotic factor (Edlich 2018). Our results further demonstrated that miR-125b overexpression efficiently attenuated TACinduced cardiac function injury, including myocardial tissue fibrosis. Meanwhile, miR-125b overexpression significantly reversed the TAC-induced effect on the expression of apoptosis-related proteins including C-caspase 3 , Bax and Bcl-2, suggesting that miR-125b overexpression significantly inhibited TAC-induced cardiomyocyte apoptosis in HF mice.

BAK1, a pro-apoptotic factor, has been reported to act as the direct target of miRNAs, is involved in cardiac apoptosis and MI (Su et al. 2020; Zhou et al. 2019). In the heart, BAK1 was reported to induce cardiomyocyte and myocardial I/R-mediated apoptosis (Bayoumi et al. 2018b). Recent studies have shown that BAK1 was regulated by miR-125b in cancer cells and neural crest cells (Wang et al. 2013; Chen et al. 2015). It has also been demonstrated that miR-125b-5p protected the heart from AMI by repressing BAK1 expression in cardiomyocytes. In our study, the bioinformatic analysis showed that there were complementary bases between miR-125b and BAK1. Moreover, luciferase analysis and miR-125b overexpression assay also showed that BAK1 was a direct target of miR-26a. Reportedly, BAK1 was significantly increased in mouse hearts during $I / R$ injury and in cardiomyocytes subjected to I/R simulation (Bayoumi et al. 2018b). We also demonstrated that BAK1 expression in TAC-induced HF mice was significantly upregulated. Moreover, BAK1 overexpression significantly reversed agomir-125b-induced protective effect on cardiac function injury, cardiomyocyte apoptosis and the expression of apoptosis-related markers C-caspase3, Bax and Bcl-2.

\section{Conclusion}

In conclusion, our results demonstrated that miR-125b overexpression efficiently alleviated HF through inhibiting cardiomyocyte apoptosis by targeting BAK1, suggesting that miR-125b/BAK1 axis might be a potential target for the treatment of HF. 


\section{Supplementary Information}

The online version contains supplementary material available at https://doi. org/10.1186/s10020-021-00328-w.

Additional file 1: Fig. S1. MiR-125b overexpression alleviated the expression of Col I and promoted col III expression in TAC-induced myocardial tissues. ${ }^{*} \mathrm{p}<0.05$.

Additional file 2: Fig. S2. MiR-125b overexpression efficiently inhibited cardiomyocyte apoptosis in HF mice. Immunofluorescence staining (A) and Western Blot of isolated myocytes (B) for both Cas-3 and myocyte markers were performed to conclude that these apoptotic markers were from cardiac myocytes. ${ }^{*} \mathrm{p}<0.05$.

Additional file 3: Fig. S3. BAK1 overexpression reversed the effects of mir-125b on Col I and col III expression in TAC-induced myocardial tissues. ${ }^{*} p<0.05$.

Additional file 4: Fig. S4. BAK1 overexpression significantly reversed agomir-125b-inhibited cardiomyocyte apoptosis in HF mice. Immunofluorescence staining (A) and Western Blot of isolated myocytes (B) for both Cas-3 and myocyte marker were performed to conclude that these apoptotic markers came from cardiac myocytes. ${ }^{*} p<0.05$.

\section{Acknowledgements}

Not applicable.

\section{Authors' contributions}

BZ, SM, XL, SL: study concepts, literature research, clinical studies, data analysis, experimental studies, manuscript writing and review; HZ: study design, literature research, experimental studies and manuscript editing; YG, WL: definition of intellectual content, clinical studies, data acquisition and statistical analysis; LF: data acquisition, manuscript preparation and data analysis; CL, PW: data acquisition and statistical analysis. All authors read and approved the final manuscript.

\section{Funding}

This work was supported by the National Nature Science Foundation of China (Grant No. 81960315, 31760294), the Science and Technology Fund of Guizhou Provincial Health Department (Grant No. [2018] 5779-39, QKHJ (2016)1128) and the Fund of Guiyang Science and Technology Department (Grant No. ZKH (2019)9-14-6).

\section{Availability of data and materials}

The data that support the findings of this study are available on request from the Corresponding authors. The data are not publicly available due to their containing information that could compromise the privacy of research participants.

\section{Declarations}

\section{Ethics approval and consent to participate}

Informed consent was obtained from all individual participants included in the study. All producers were approved by the Guizhou Medical University Ethics Committee.

\section{Consent for publication}

Not applicable.

\section{Competing interests}

All other authors have no conflicts of interest. We declare that we do not have any commercial or associative interest that represents a conflict of interest in connection with the work submitted.

\section{Author details}

'Guizhou Medical University, No. 9 Beijing Road, Yunyan District, Guiyang, Guizhou 550004, People's Republic of China. ${ }^{2}$ Department of Ultrasound Medicine, The Affiliated Hospital of Guizhou Medical University, No. 28 Guiyi Street, Yunyan District, Guiyang, Guizhou 550004, People's Republic of China.
${ }^{3}$ Department of Cardiology, The Second Affiliated Hospital of Guizhou University of Traditional Chinese Medicine, Guiyang, Guizhou 550004, People's Republic of China. ${ }^{4}$ Departmentof Clinical Research Centre, The Affiliated Hospital of Guizhou Medical University, Guiyang, Guizhou 550004, People's Republic of China. ${ }^{5}$ Department of Cardiology, The Affiliated Baiyun Hospital of Guizhou Medical University, Guiyang City, Guizhou 550014, People's Republic of China.

Received: 23 October 2020 Accepted: 10 June 2021

Published online: 08 July 2021

\section{References}

Abdo AS. Hospital management of acute decompensated heart failure. Am J Med Sci. 2017;353(3):265-74.

Batool A, Wang YQ, Hao XX, Chen SR. A miR-125b/CSF1-CX3CL1/tumor-associated macrophage recruitment axis controls testicular germ cell tumor growth. Cell Death Dis. 2018;9(10):962.

Bayoumi AS, Park KM, Wang Y, Teoh JP, Aonuma T, Tang Y, et al. A carvedilolresponsive microRNA, miR-125b-5p protects the heart from acute myocardial infarction by repressing pro-apoptotic bak 1 and klf13 in cardiomyocytes. J Mol Cell Cardiol. 2018a;114:72-82.

Bie ZD, Sun LY, Geng CL, Meng QG, Lin XJ, Wang YF, et al. MiR-125b regulates SFRP5 expression to promote growth and activation of cardiac fibroblasts. Cell Biol Int. 2016:40(11):1224-34.

Burchfield JS, Xie M, Hill JA. Pathological ventricular remodeling: mechanisms: part 1 of 2. Circulation. 2013;128(4):388-400.

Butler J, Fonarow GC, Zile MR, Lam CS, Roessig L, Schelbert EB, et al. Developing therapies for heart failure with preserved ejection fraction: current state and future directions. JACC Heart Failure. 2014;2(2):97-112.

Cao W, Qian G, Luo W, Liu X, Pu Y, Hu G, et al. miR-125b is downregulated in systemic lupus erythematosus patients and inhibits autophagy by targeting UVRAG. Biomed Pharmacotherapy. 2018;99:791-7.

Chen X, Liu J, Feng WK, Wu X, Chen SY. MiR-125b protects against ethanolinduced apoptosis in neural crest cells and mouse embryos by targeting Bak 1 and PUMA. Exp Neurol. 2015;271:104-11.

Davis J, Molkentin JD. Myofibroblasts: trust your heart and let fate decide. J Mol Cell Cardiol. 2014;70:9-18.

Deng H, Ouyang W, Zhang L, Xiao X, Huang Z, Zhu W. LncRNA GASL1 is downregulated in chronic heart failure and regulates cardiomyocyte apoptosis. Cell Mol Biol Lett. 2019;24:41.

Edlich F. BCL-2 proteins and apoptosis: recent insights and unknowns. Biochem Biophys Res Commun. 2018;500(1):26-34.

González A, Schelbert EB, Díez J, Butler J. Myocardial interstitial fibrosis in heart failure: biological and translational perspectives. J Am Coll Cardiol. 2018;71(15):1696-706.

Hanna A, Shinde A, Li R, Alex L, Humeres C, Balasubramanian P, et al. Collagen denaturation in the infarcted myocardium involves temporally distinct effects of MT1-MMP-dependent proteolysis and mechanical tension. Matrix Biol J Int Soc Matrix Biol. 2021. https://doi.org/10.1016/j.matbio. 2021.05.005.

Jiang D, Wei X, Zhang X, Liu Y, Zhang Y, Chen K, et al. IRF8 suppresses pathological cardiac remodelling by inhibiting calcineurin signalling. Nat Commun. 2014;5:3303.

Jin D, Ojcius DM, Sun D, Dong H, Luo Y, Mao Y, et al. Leptospira interrogans induces apoptosis in macrophages via caspase-8- and caspase-3-dependent pathways. Infect Immun. 2009;77(2):799-809.

Jugdutt BI, Mohammad I, et al. Combined captopril and isosorbide dinitrate during healing after myocardial infarction: effect on ventricular remodeling, function, mass and collagen - ScienceDirect. J Am Coll Cardiol. 1995;25(5):1089-96.

Lakatta EG. Age-associated cardiovascular changes in health: impact on cardiovascular disease in older persons. Heart Fail Rev. 2002;7(1):29-49.

Li LN, Xiao T, Yi HM, Zheng Z, Qu JQ, Huang W, et al. MiR-125b increases nasopharyngeal carcinoma radioresistance by targeting A20/NF-KB signaling pathway. Mol Cancer Ther. 2017;16(10):2094-106.

Li W, Kennedy D, Shao Z, Wang X, Kamdar AK, Weber M, et al. Paraoxonase 2 prevents the development of heart failure. Free Radical Biol Med. 2018a;121:117-26. 
Li X, Tang X, Lu J, Yuan S. Therapeutic inhibition of galectin-3 improves cardiomyocyte apoptosis and survival during heart failure. Mol Med Rep. 2018b;17(3):4106-12.

Liu J, Guo B, Chen Z, Wang N, lacovino M, Cheng J, et al. miR-125b promotes MLL-AF9-driven murine acute myeloid leukemia involving a VEGFA-mediated non-cell-intrinsic mechanism. Blood. 2017;129(11):1491-502.

Liu BL, Cheng M, Hu S, Wang S, Wang L, Tu X, et al. Overexpression of miR142-3p improves mitochondrial function in cardiac hypertrophy. Biomed Pharmacotherapy. 2018;108:1347-56.

Martinelli MV, Bösch C, Signorell J, Mohacsi P. Advanced heart failure-treatment options beyond medical management. Therapeutische Umschau Revue Therapeutique. 2011;68(12):715-23.

Mazurek JA, Jessup M. Understanding heart failure. Heart Fail Clin. 2017;13(1):1-19.

McMurray JJ, Pfeffer MA. Heart failure. Lancet (london, England). 2005;365(9474):1877-89.

Metra M, Teerlink JR. Heart failure. Lancet (london, England). 2017:390(10106):1981-95

Nagpal V, Rai R, Place AT, Murphy SB, Verma SK, Ghosh AK, et al. MiR-125b is critical for fibroblast-to-myofibroblast transition and cardiac fibrosis. Circulation. 2016;133(3):291-301.

Qiao L, Hu S, Liu S, Zhang H, Ma H, Huang K, et al. microRNA-21-5p dysregulation in exosomes derived from heart failure patients impairs regenerative potential. J Clin Investig. 2019;129(6):2237-50.

Schelbert EB, Fonarow GC, Bonow RO, Butler J, Gheorghiade M. Therapeutic targets in heart failure: refocusing on the myocardial interstitium. J Am Coll Cardiol. 2014;63(21):2188-98.

Shah P, Bristow MR, Port JD. MicroRNAs in heart failure, cardiac transplantation, and myocardial recovery: biomarkers with therapeutic potential. Curr Heart Fail Rep. 2017;14(6):454-64.

Su X, Lv L, Li Y, Fang R, Yang R, Li C, et al. IncRNA MIRF promotes cardiac apoptosis through the miR-26a-Bak1 axis. Mol Therapy Nucleic Acids. 2020;20:841-50

Tanai E, Frantz S. Pathophysiology of Heart Failure. Compr Physiol. 2015:6(1):187-214.

Vegter EL, van der Meer P, de Windt LJ, Pinto YM, Voors AA. MicroRNAs in heart failure: from biomarker to target for therapy. Eur J Heart Fail. 2016;18(5):457-68.

Verjans R, Peters T, Beaumont FJ, van Leeuwen R, van Herwaarden T, Verhesen W, et al. MicroRNA-221/222 family counteracts myocardial fibrosis in pressure overload-induced heart failure. Hypertension Dallas Tex. 2018;71(2):280-8.

Vishnoi A, Rani S. MiRNA biogenesis and regulation of diseases: an overview. Methods Mol Biol (clifton, NJ). 2017;1509:1-10.
Wang YD, Cai N, Wu XL, Cao HZ, Xie LL, Zheng PS. OCT4 promotes tumorigenesis and inhibits apoptosis of cervical cancer cells by miR-125b/BAK1 pathway. Cell Death Dis. 2013;4(8):e760.

Wang K, Long B, Liu F, Wang JX, Liu CY, Zhao B, et al. A circular RNA protects the heart from pathological hypertrophy and heart failure by targeting miR-223. Eur Heart J. 2016;37(33):2602-11.

Xu D, Zhao Y, Weng X, Lu Y, Li W, Tang K, et al. Novel role of mitochondrial GTPases 1 in pathological cardiac hypertrophy. J Mol Cell Cardiol. 2019;128:105-16

Yan Z, Xu T, An Z, Hu Y, Chen W, Ma J, et al. Costunolide induces mitochondriamediated apoptosis in human gastric adenocarcinoma BGC-823 cells. BMC Complement Altern Med. 2019;19(1):151.

Yang J, Savvatis K, Kang JS, Fan P, Zhong H, Schwartz K, et al. Targeting LOXL2 for cardiac interstitial fibrosis and heart failure treatment. Nat Commun. 2016;7:13710.

Yang X, Tang S, Li D, Yu X, Wang F, Xiao X. DIDS inhibits overexpression BAK1induced mitochondrial apoptosis through GSK3 $\beta / \beta$-catenin signaling pathway. J Cell Physiol. 2018;233(6):5070-7.

Yang W, Han Y, Yang C, Chen Y, Zhao W, Su X, et al. MicroRNA-19b-1 reverses ischaemia-induced heart failure by inhibiting cardiomyocyte apoptosis and targeting Bcl2 111/BIM. Heart Vessels. 2019;34(7):1221-9.

Zhao J, Zeng Z, Yan L, Ji LJ, Luo T, Bin JP, et al. Echocardiographic evaluation of pressure overload-induced cardiac remodeling in mice using different ultrasound machines. Nan fang yi ke da xue xue bao J South Med Univ. 2011:31(3):443-7.

Zhao Y, Ponnusamy M, Liu C, Tian J, Dong Y, Gao J, et al. MiR-485-5p modulates mitochondrial fission through targeting mitochondrial anchored protein ligase in cardiac hypertrophy. Biochim Biophys Acta. 2017:1863(11):2871-81.

Zhou HC, Fang JH, Shang LR, Zhang ZJ, Sang Y, Xu L, et al. MicroRNAs miR$125 \mathrm{~b}$ and miR-100 suppress metastasis of hepatocellular carcinoma by disrupting the formation of vessels that encapsulate tumour clusters. J Pathol. 2016;240(4):450-60.

Zhou Y, Richards A, Wang P. MicroRNA-221 is cardioprotective and anti-fibrotic in a rat model of myocardial infarction. Mol Therapy Nucleic Acids. 2019;17:185-97.

\section{Publisher's Note}

Springer Nature remains neutral with regard to jurisdictional claims in published maps and institutional affiliations.
Ready to submit your research? Choose BMC and benefit from:

- fast, convenient online submission

- thorough peer review by experienced researchers in your field

- rapid publication on acceptance

- support for research data, including large and complex data types

- gold Open Access which fosters wider collaboration and increased citations

- maximum visibility for your research: over $100 \mathrm{M}$ website views per year

At BMC, research is always in progress.

Learn more biomedcentral.com/submissions 